\title{
Identification and Vibration Attenuation for the Parallel Robot Par2
}

\author{
Luiz R. Douat, Isabelle Queinnec, Germain Garcia, Micaël Michelin, François Pierrot and Sophie Tarbouriech
}

\begin{abstract}
Par2 is a two-degree-of-freedom parallel robot designed for high-speed and high-accuracy industrial pick-andplace operation tasks. As a result of the high acceleration trajectories, the end-effector undergoes some undesirable vibrations after reaching the stop positions, compromising its precision and leading therefore to an increase in the operation cycle time. Accelerometer sensors placed on the end-effector and piezoelectric patch actuators wrapped around the robot arms are employed in order to actively reduce these vibrations in a non-collocated closed-loop setting. After submitting the robot to an identification procedure, the obtained nominal model is used to synthesize a reduced order controller with the $\mathscr{H}_{\infty}$ Loop Shaping technique. Performance analysis as well as simulation and experimental results exhibit that vibration reduction is achieved around the nominal operating point, but fails for some extreme operating points, due to high control efforts. An antiwindup strategy is then employed to deal with the saturation of the actuator, which allows to achieve vibration attenuation on the whole operation domain, for a given configuration of the robot at the stop point.
\end{abstract}

Index Terms-High-speed parallel robotics, robust control, vibration attenuation, piezoelectric actuators, saturation, antiwindup.

\section{INTRODUCTION}

In the context of industrial handling and assembly tasks, the increasing demand for productivity imposes more and more strict requirements in terms of production cycle times. Lighter than their serial counterparts, parallel robots [24] are able to accomplish the desired trajectories faster and with a better precision. For pick-and-place operations, these higher trajectory accelerations demand correspondingly higher decelerations each time the end-effector reaches some stop position, giving rise to undesirable vibrations and therefore impairing the positioning accuracy. The gain obtained in reducing the trajectory time by using higher accelerations is thus countered by an increase in the residual vibration levels after reaching the stop positions.

For minimizing these vibrations, different measures could be undertaken:

1) Improvement of the robot design (light but stiff robot links) [5], [22];

L.R. Douat, I. Queinnec, G. Garcia and S. Tarbouriech are with CNRS, LAAS, 7 avenue du colonel Roche, BP54200, F-31031 Toulouse Cedex 4, France and also with Univ de Toulouse, INSA, LAAS, F-31400 Toulouse, France. (isabelle.queinnec@laas.fr).

M. Michelin is with Tecnalia, Rond-point Benjamin Franklin, CS 39521, 34960 Montpellier, Cedex 2, France.

F. Pierrot is with Université Montpellier 2, LIRMM, UMR 5506 - CC 477, 161 rue Ada, 34095 Montpellier, Cedex 5, France, where all experiments with Par2 were performed.
2) Optimization of the pick-and-place trajectory, avoiding inflection points in the higher successive position derivatives [6], [11];

3) Active vibration control during the trajectory by means of the motor magnetic moments [7], [21];

4) Active vibration control during the trajectory by means of piezoelectric actuators [13], [14]. This case is of particular interest when the vibrations may induce hazardous consequences to the robot during the trajectory;

5) Active vibration control at the stop positions by means of piezoelectric actuators [15], [25], [29]. According to the type and placement of the sensors and actuators in the robot, two different architectures can be characterized: collocated and non-collocated. In the non-collocated case [30], [34], addressed in this paper, different kinds of sensors can also be used, as for instance accelerometers [1], generally allowing better performances [37].
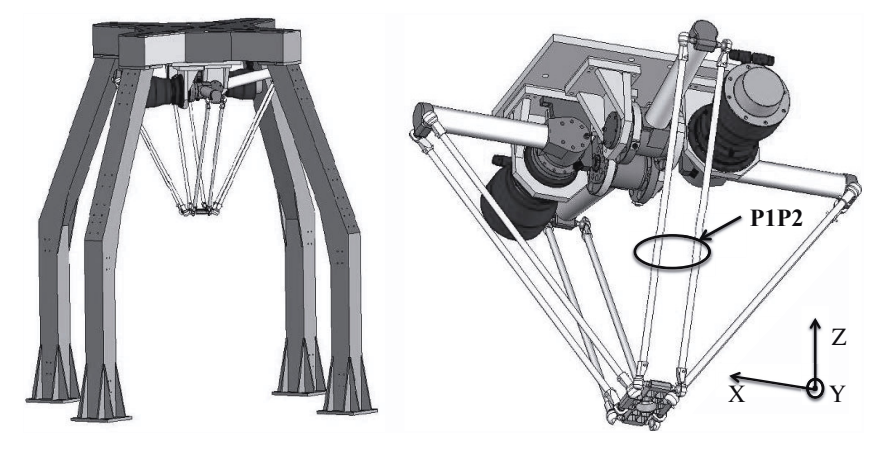

Fig. 1. Parallel robot - Par2

Par2, depicted in Figure 1, is one such fast pick-andplace two-degree-of-freedom parallel robot [3]. Despite its very good stiffness properties, there are still some remaining vibrations showing up at the end of each trajectory, which are measured by means of three orthogonally oriented accelerometers (in $X, Y$ and $Z$ directions) placed on the end-effector. Given that these vibrations hinder the accurate positioning of the end-effector at the stop positions, it is at these precise points that active control is required. Following the strategy adopted in [1], piezo-actuator patches are glued all along and around the arms of Par2 in order to attenuate the residual vibrations by means of the controlled deformation of the arms (section II). The control objective is then to reject disturbances, seen as the residual vibrations coming up just after the arrival of the end-effector at a stop position, for various trajectories and load conditions. 
In order to synthesize a control law, it is essential to have first a representative model of the plant. Piezoelectric devices generally exhibit nonlinear response behavior involving hysteresis, drift, creep, and/or time delay [19], which may be critical in certain applications of high-precision positioning of nano-devices. In the current work, however, we are mainly concerned with the vibration response of the piezo-elements further used to compensate for the vibrations induced by the motion of the robot. Set as a black box connecting the piezoelectric voltage (system input) to the measured endeffector acceleration (system output), a system identification procedure is performed using state subspace techniques [20], leading to the production of a nominal model of the system [8] (section III).

Aiming at synthesizing a robust controller, capable of attenuating the vibrations generated at different operating points (trajectories and load conditions), the $\mathscr{H}_{\infty}$ Loop Shaping strategy is employed (section IV). It differs from previously published papers from the authors on the subject, where a mixed sensitivity $H_{\infty}$ design strategy was considered [9], [10]. The current strategy is adopted here to benefit from its easy implementation with robust and easily tunable filters.

For some operating points kept away from the nominal point, saturation of the actuator may however induce a nasty effect, from loss of performance to loss of stability. Then, an anti-windup compensator strategy may be addressed as an additional control loop on the closed-loop system, active only when saturation occurs. Many different anti-windup design strategies have been addressed in the literature [32], [39]. We consider here a direct linear anti-windup compensator design, and we restrict to the case of a simple static anti-windup gain which acts on the output of the robust controller previously designed (section $\mathrm{V}$ ).

At each step of the whole control design procedure, simulations and experimental results are proposed to exhibit the potential interest of the approach proposed in this paper.

\section{Problem StATEMENT}

The manipulator end-effector trajectories (dash-dotted representation in Figure 2), constrained to the $x z$ plane between stop positions Pos1 $(x=0.35 m, z=-0.925 m)$ and Pos2 $(x=-0.35 m, z=-0.925 m)$, are commanded by the so-called active arms (those directly connected to the motors, Figure 1) while the passive ones hinder, as much as possible, the perpendicular motions.

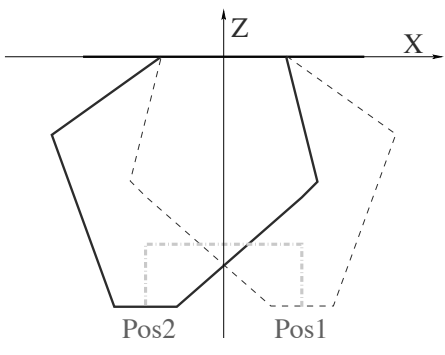

Fig. 2. Stop positions
The piezo-actuator patches are glued on those arms (Figure 3 ), and are labelled with letters $A$ and $P$, given accordingly to the active or passive characteristic of the arm. They are PZT elements (PI, DuraAct P-876.A12) whose deformation can be considered linear with respect to an input voltage signal ranging from 0 to $400 \mathrm{~V}$. Each piezo-actuator input voltage signal is supplied by means of a high-voltage amplifier (Walcher Electronik, HVA-400-100-ISO) with bandwidth of 8 $k H z$, that amplifies the $-10 \mathrm{~V}$ to $10 \mathrm{~V}$ control signal sent by the control unit (Adept, sMI6).

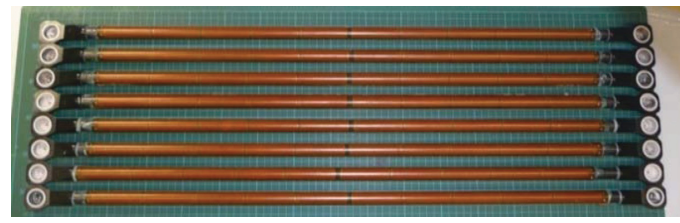

Fig. 3. Par2s arms equipped with the piezoelectric patches

Despite presenting vibrations during the whole pick-andplace trajectory, their effects are problematic only when amplified by the high decelerations on the arrival at a stop position, overly exciting some modes of the robot arms and translating into loss of end-effector precision. These amplified vibrations at the stop positions are called the disturbances to our system. The accelerometers (BrüelKjaer, DeltaTron 4506) employed to measure these vibrations are able to provide linear measures up to $700 \mathrm{~m} / \mathrm{s}^{2}(70 \mathrm{~g})$ with a sensitivity of $100 \mathrm{mV} / \mathrm{g}$ and frequencies between $0.3 \mathrm{~Hz}$ and $3 \mathrm{kHz}$. Before sending them to the control unit, the accelerometer signals are filtered by a charge amplifier (Endevco, Model 133) of $100 \mathrm{kHz}$ bandwidth and adjustable cut-off frequency.

The pick-and-place motions of the robot (from Pos1 to Pos2 and vice versa) can be accomplished for many different operating points. Each operating point is characterized by a load on the end-effector (varying from empty to $3.5 \mathrm{~kg}$ ) and a particular trajectory peak acceleration (varying from $10 \mathrm{~g}$ to $30 \mathrm{~g}$ ). Considering that the robot motor currents are limited to $30 \mathrm{~A}$, the higher is the load on the end-effector, the lower is the maximum acceleration allowed for a trajectory. The operating points taken into account in this work, for which the residual vibrations have to be reduced, are depicted in Figure 4, where the upper circles represent the maximal pick acceleration for each load condition.

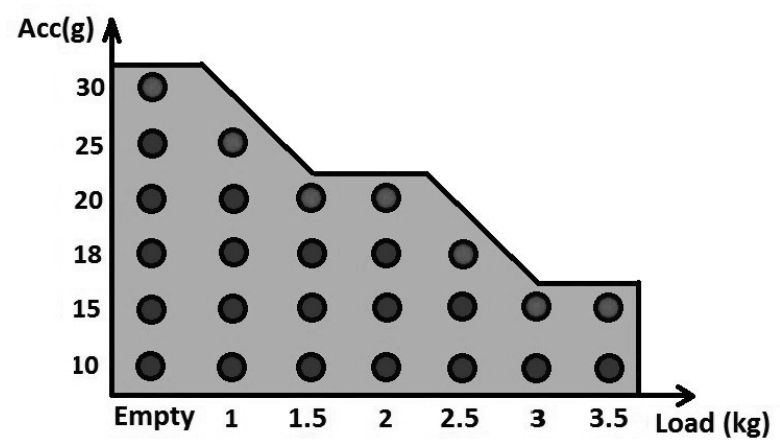

Fig. 4. Operating points - load conditions and trajectories 
We consider in this work the case where the piezo control is activated immediately after arriving at the stop position Pos2 (the residual vibrations observed at the stop positions Pos1 and Pos 2 are symmetrical and, thus, only Pos 2 is considered here). For the control law synthesis, we first need to identify a nominal open-loop dynamical system which connects the piezo-actuator to the accelerometer. Such a model is associated to a particular nominal operating point. We then want to synthesize a robust control law, that is, a controller able to reduce the vibrations not only for the nominal operating point, but also for the largest set of uncertain model representations around this point. We choose a $2.5 \mathrm{~kg}$ load with a $18 \mathrm{~g}$ trajectory to be our nominal operating point and want to verify the efficacy of its associated robust controller over the whole space of selected operating points (Figure 4).

It was experimentally verified that, due to a much lower damping factor, the residual vibrations in all desired operating points are much more substantial in the $Y$ direction. This phenomenon is illustrated for the nominal operating point in Figure 5, in the frequency domain, and in Figure 6, in the time domain. Two experiments are plotted, dist 1 and dist 2, to illustrate that they are reproducible. Figure 5 exhibits a low damped flexible mode around $21.7 \mathrm{~Hz}$ in the $Y$ direction. In Figure 6 , the disturbances in the $Y$ direction between $0.01 \mathrm{~s}$ and $1 s$ are far less damped than the disturbances in the other two directions.
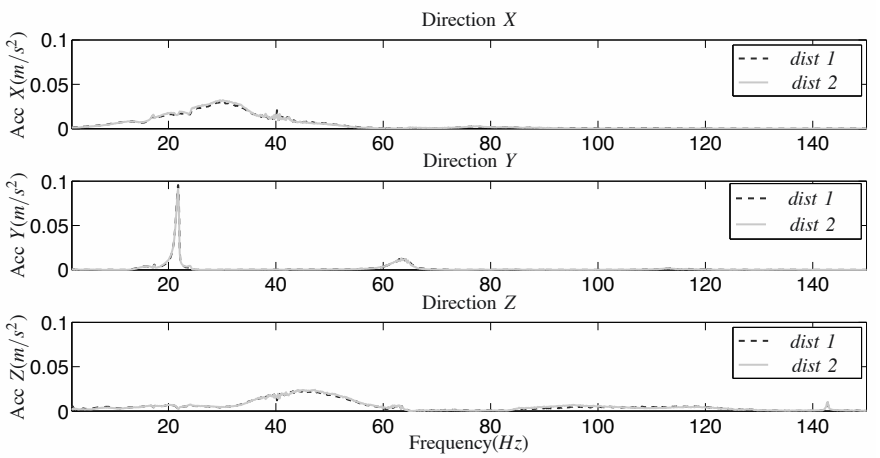

Fig. 5. Disturbances dist 1 and dist 2 - Nominal operating point - Frequency domain

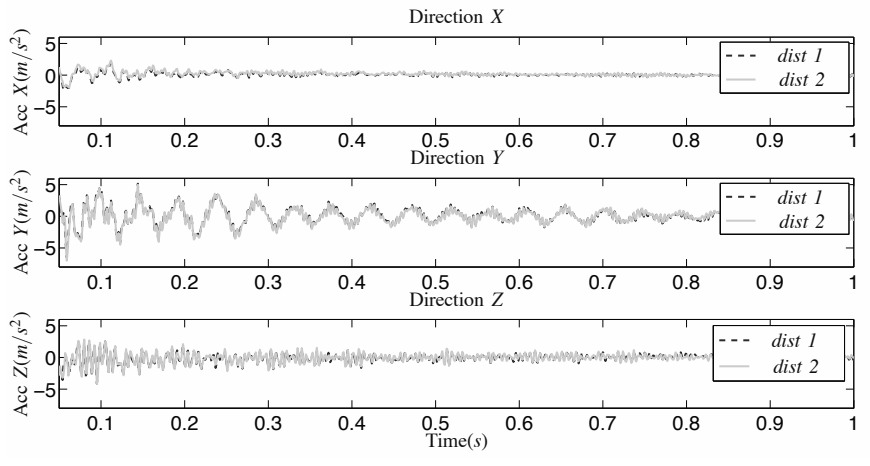

Fig. 6. Disturbances dist 1 and dist 2 - Nominal operating point - Time domain

To control the acceleration in the $Y$ direction, it is rec- ommended to manipulate the piezo-element patches on the passive arms (labelled with $P$ ), which are exactly provided to limit those off-line vibrations. Then in order to remain as simple as possible, rather than to consider a multiple input system with one input associated with each passive piezoactuated arm, we prefer to select a pair of passive piezoactuated arms (namely $P 1 P 2$ in Figure 1), jointly and equally actuating and from now on considered as a single element, to be our system input. We then consider a SISO control problem and, moreover, we keep free the other piezo-actuators for a potential future extension of the approach to the full MIMO control problem.

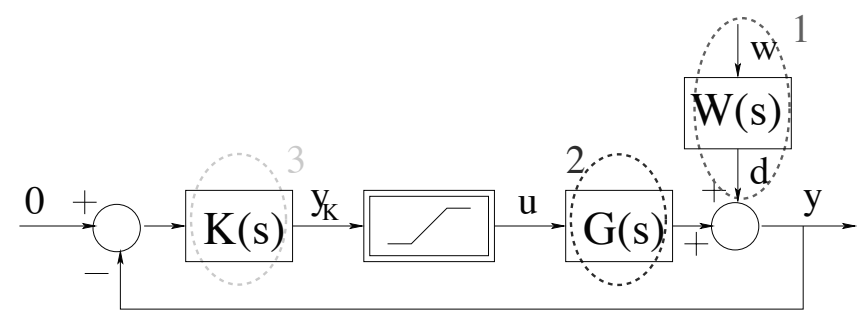

Fig. 7. Function block representation of the closed-loop system

According to the system representation of Figure 7 , this work can be initially divided into 3 steps. The first step, briefly described above and presented in detail in [8], is the characterization of the frequency spectra of the disturbance signal $d$ for the nominal operating point. The second step concerns the open-loop identification of the nominal model $G$ from the piezo-actuator input voltage $u$ to the accelerometer in the $Y$ direction (section III). The third set addresses the design problem of a robust controller $K$ capable of attenuating the vibrations for the nominal operating point as well as for all operating points represented in Figure 4 (section IV). It is initially done without taking explicitly into account the saturation of the voltage delivered to the piezo-actuator. Then, the influence of this nonlinear element in the closed-loop may be further studied (section V).

\section{IDENTIFICATION}

Dynamical model production can be either analytic or blackbox based. An analytic model is obtained based on physical principles and allows a better comprehension of what is happening inside the model. Its main disadvantages are the often strenuous conceptual phase and the need of further experimental parameter validation (data updating). If the dynamical model is to be used only for control purposes, a fast-to-obtain and easy-to-validate alternative is to employ black-box system identification techniques. These identification techniques deal with the problem of building mathematical models of dynamical systems based only on its input-output data [20]. The validation of these models is often done by the comparison in the frequency domain of the estimated model with a nonparametric model obtained from the same input-output data. A typically used non-parametric model in the frequency domain is called ETFE (Empirical Transfer Function Estimate), given by the division of the discrete Fourier transforms of the output and input vector signals. 
We can classify the identification techniques into classical methods and methods based on subspaces. The works of [20] and [36] show that subspace identification methods allow an easy extraction of good quality models. For that reason, the open-loop models relating the piezo-actuator input voltage to the accelerometer output of Par 2 are identified based on one of these subspace methods. The identification procedure is performed twice, giving rise to two models: one used for control synthesis and another for validation of the obtained controller in computer simulations.

\section{A. Subspace identification methods}

Subspace identification emerged from the association of the multivariable statistical analyses methods (stochastic realization) from the 30's and modern numerical linear algebra tools like QR and SVD decompositions [16], [17]. Contrarily to classical parameter identification methods as ARMAX and Box-Jenkins [20], subspace methods allow to obtain a statespace system representation based on the estimation of certain subspaces from matrices formed with the input and output experimental data vectors. Some advantages from subspace methods over the classical ones are:

- Only the desired (expected) system order for the statespace model must be provided as an entry parameter. This unique-parameter feature makes subspace methods easier to employ than their counterpart methods (classical parametric methods of equivalent complexity, where the polynomial orders have to be specified, usually require from 3 to 5 tuning parameters);

- There are no nonlinear optimization problems. Subspace methods are based on subspace matrices projections without any parametric optimization procedure involved, avoiding problems like local-minima and non-convexity;

- The method is directly expandable to multivariable systems and without the drawbacks of classical methods applied to MIMO systems which often give ill-conditioned models and are usually very time demanding [4].

There exist many different subspace identification algorithms: MOESP [35], IVM [38], CVA [18], N4SID [28], etc. In this work the N4SID (Numerical algorithm For Subspace IDentification) algorithm was employed because of its proven applicability to the identification of vibration system models [31], [26], [8].

In the N4SID algorithm, an extended observability matrix of a system with state-space representation in some base $T$ is estimated through the input-output data vectors. This observability matrix allows the extraction of a Kalman filter state sequence which is then used to obtain, by means of the solution of a least square problem, an estimate of the statespace matrices of the original system in the same base $T$.

\section{B. Open-loop piezo-actuator identification}

For the identification procedure, a chosen input voltage signal excited the resonant modes of the piezo-actuator $P 1 P 2$. The corresponding time response vibration was then stored for the accelerometer measurements in the $Y$ direction. These input and output data vectors were pre-processed and used to build a mathematical description of the piezo-actuator dynamical behaviour, by means of the N4SID subspace identification algorithm. The identified model of the piezo-actuator did not depend on the peak acceleration as the identification experiments were performed with the end-effector initially at rest at the position Pos2. On the other hand, it depended on the load at the end-effector. We considered here the end-effector with a nominal load of $2.5 \mathrm{~kg}$. The experimental identification set-up is summarized in table I.

TABLE I

EXPERIMENTAL IDENTIFICATION SET-UP

\begin{tabular}{l|l}
\hline Signal type & linear CHIRP \\
\hline Signal sweep range & 0 to $150 \mathrm{~Hz}$ \\
\hline Signal duration & $100 \mathrm{~s}$ \\
\hline Sampling rate & $1 \mathrm{kHz}$ \\
\hline Low-pass analogic & $500 \mathrm{~Hz}$ \\
cut-off frequency & (Nyquist frequency) \\
\hline $\begin{array}{l}\text { Low-pass digital } \\
\text { cut-off frequency }\end{array}$ & $150 \mathrm{~Hz}$ \\
\hline
\end{tabular}

During the identification steps, a sampling rate of $1 \mathrm{kHz}$ was chosen after verification that the initially adopted $2 \mathrm{kHz}$ sampling rate led to stable but ill conditioned models (poles very close to each other near the real value 1 in the unit circle). According to the identification experiments performed in [1] and [8], a chirp signal was used as input excitation. It was experimentally verified that the highest main frequency modes excited in the $Y$ direction for all considered operating points (Figure 4) did not surpass by far the limit of $30 \mathrm{~Hz}$. For that reason the chirp signal used was empirically chosen to sweep linearly a five times larger spectrum (from 0 up to $150 \mathrm{~Hz}$ ) during $100 \mathrm{~s}$ (sufficient to minimize the influence of the sweeping transitory effects [20]). The output acceleration signals were analogically low-pass filtered by the charge amplifier with a cut-off frequency set to $500 \mathrm{~Hz}$ (Nyquist frequency) before being stored. Despite the analogical filtering process, the noise levels verified in the output data were still considerable. Therefore, a digital 5th order low-pass Butterworth filter with $150 \mathrm{~Hz}$ cut-off frequency was applied to the input-output stored data, shown in Figure 8.

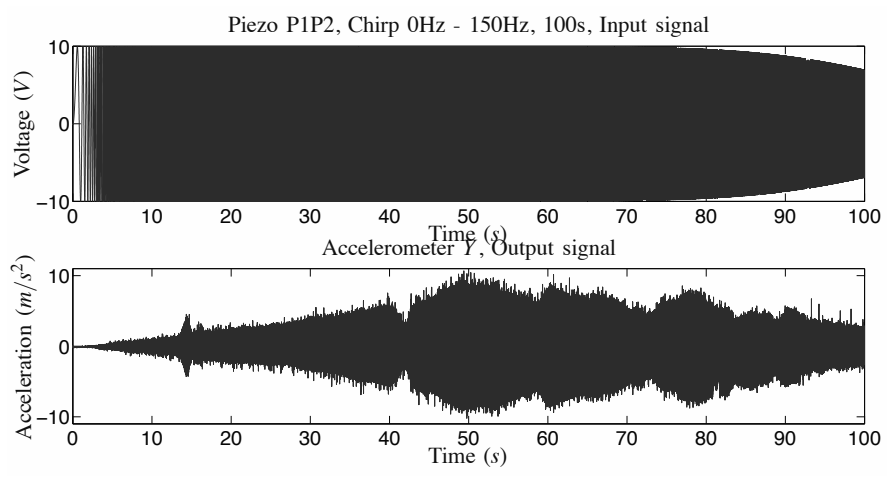

Fig. 8. Data for mod ctrl $2.5 \mathrm{~kg}$ - Input and output signals

The procedure was applied twice to obtain two input-output data vectors. It has been checked that associate non-parametric 
frequency models show a good reproducibility between the two experiments. They were then used to build two models, initially named mod ini ctrl $2.5 \mathrm{~kg}$ and mod ini sim $2.5 \mathrm{~kg}$. These models were further reduced and became $\bmod \mathrm{ctrl} 2.5 \mathrm{~kg}$, used for control synthesis purposes, and $\bmod \operatorname{sim} 2.5 \mathrm{~kg}$, employed for validating the obtained controller in numerical simulations.

Let us consider the first set of input-output data. By using the N4SID algorithm with different desired state-space model orders, the obtained candidate mod ini $\mathrm{ctrl} 2.5 \mathrm{~kg}$ models can be seen in Figure 9 with also the non-parametric model directly issued from the experimental data (NP model). Both amplitude and phase are represented in this figure. Nevertheless, the control technique adopted in this work is based on a worst case amplitude criterion without any consideration to the phase. This reason motivated our choice to present, from now on, only the harmonic gain amplitudes.
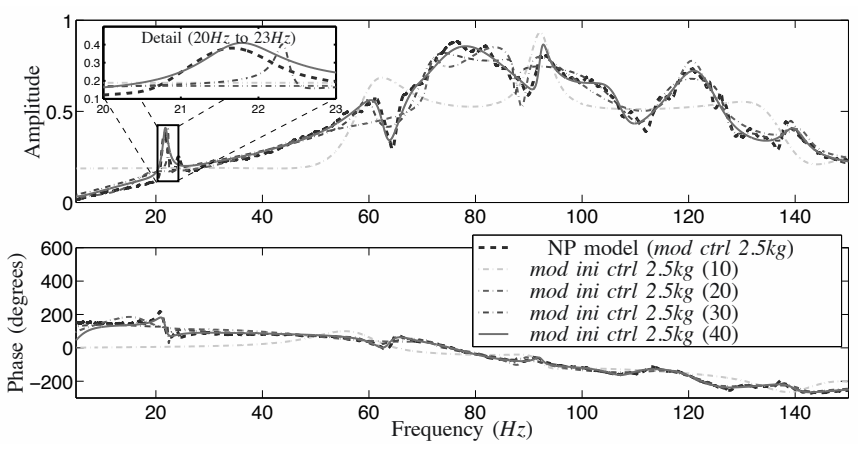

Fig. 9. Frequency domain models obtained for mod ini $\mathrm{ctrl} 2.5 \mathrm{~kg}$ with the N4SID algorithm (orders 10, 20, 30 and 40)

Figure 9 shows that the 40 order N4SID model was close to the non-parametric model (used for validation) in the frequencies of interest, being therefore the selected one. Considering the high order of the model, a reduction procedure was then performed in two steps, through techniques that preserve the main dynamical properties of the original model. Note that it is recommended to select a high-order model, then to reduce it, as an original low-order model would not focus enough on the low frequency part of the data, and would put too much emphasis to the high frequency part related to noise activity. In the first step of reduction, the high frequency modes were suppressed. Under the assumption of linearity, all the poles and zeros beyond the input signal excitation range were considered to be noisy measurements and did not reveal any information about the system. These singularities from $150 \mathrm{~Hz}$ to $500 \mathrm{~Hz}$ were thus eliminated and the model was reduced to a 22 order model. The second step consisted in rewriting the resulting system in a new base, called balanced, where the controllability and observability gramians were equal and diagonal. This realization allowed to isolate the states that less contributed to the output system dynamics. Once isolated, these states could be eliminated with, for instance, the singular perturbation model reduction method [12].

After writing the 22 order model in a balanced realization, a Hankel singular value analysis shows, in Figure 10, that the first 14 Hankel singular values correspond to $96 \%$ of the model energy. Using the singular perturbation reduction method it is therefore reduced to a 14 order model, being now called mod ctrl $2.5 \mathrm{~kg}$.

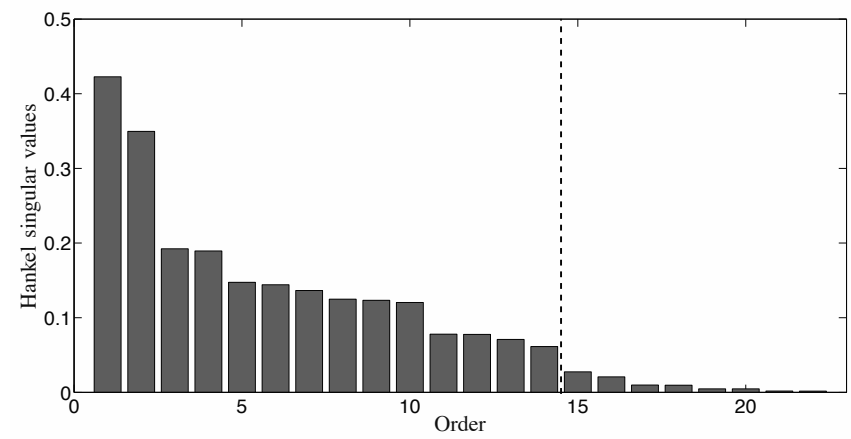

Fig. 10. Hankel singular values for the partially reduced model mod ini ctrl $2.5 \mathrm{~kg}$ of order 22

The same procedure of identification and reduction was applied also to mod ini sim $2.5 \mathrm{~kg}$ (respectively $\bmod \operatorname{sim} 2.5 \mathrm{~kg}$ ). Considering that the model for simulation shall be more informative than the model for control synthesis, the N4SID method was applied to mod ini sim $2.5 \mathrm{~kg}$ using a conservative order of 80 . The high frequency modes were suppressed and the model order was reduced to 43. After a balanced realization followed by a singular perturbation reduction method the final mod sim $2.5 \mathrm{~kg}$ model obtained was of order 30 (corresponding to $98 \%$ of the energy of the partially reduced 43 order mod ini sim $2.5 \mathrm{~kg}$ model).

The non-parametric frequency model, compared to both $\bmod \mathrm{ctrl} 2.5 \mathrm{~kg}$ (for control synthesis) and $\bmod \operatorname{sim} 2.5 \mathrm{~kg}$ (for controller validation in numerical simulations) models is shown in Figure 11.

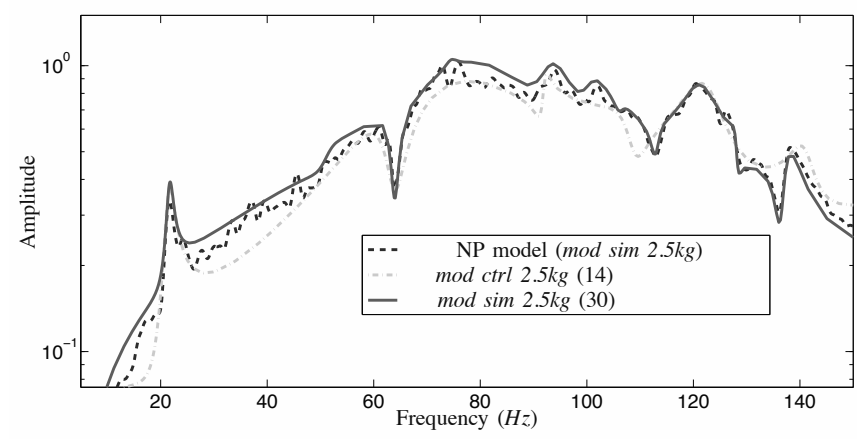

Fig. 11. Models mod ctrl 2.5kg (order 14) and mod sim 2.5kg (order 30)

The models $\bmod \mathrm{ctrl} 2.5 \mathrm{~kg}$ and $\bmod \operatorname{sim} 2.5 \mathrm{~kg}$ were identified in the discrete time domain. However, the MATLAB ${ }^{\mathbb{R}}$ function (hinfsyn from the Robust Control Toolbox) used further on to synthesize the $\mathscr{H}_{\infty}$ control law is available only for the continuous time case. This reason together with the fact that the visual analysis of poles and zeros is often easier in the continuous time domain prompted us to convert the discrete time models into the continuous time. These conversions were done by using a Tustin bilinear transformation [27]. Figure 
12, comparing the model mod ctrl $2.5 \mathrm{~kg}$ in the discrete and continuous time, shows that the approximation is valid.

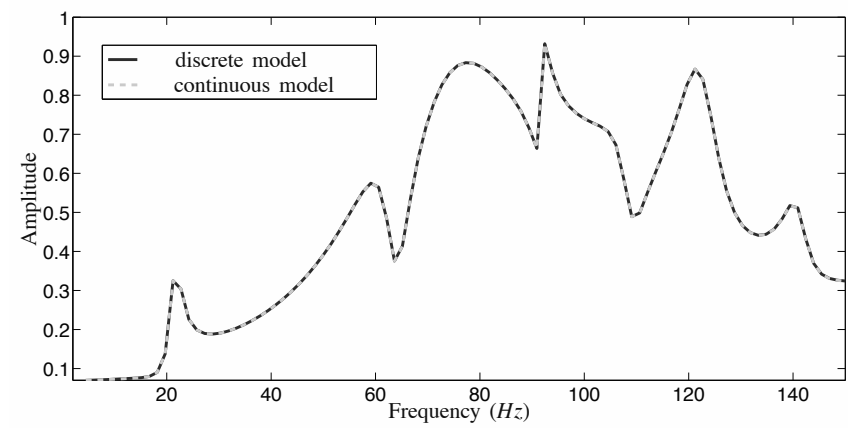

Fig. 12. Model mod ctrl $2.5 \mathrm{~kg}$ in discrete and continuous time

\section{IV. $\mathscr{H}_{\infty}$ LOOP SHAPING CONTROL}

\section{A. The $\mathscr{H}_{\infty}$ Loop Shaping Design Procedure}

Traditional loop shaping techniques are mainly based on the fact that, under certain assumptions, the magnitude of the open-loop gain can approximately determine the behavior of the associated closed-loop transfers. The uncertainties are represented acting on the normalized coprime factors of the nominal model and the robust closed-loop objectives are established in terms of (desired) shaped open-loop singular values [23], [40]. The control performance goals are usually to reduce the magnitude of the sensitivity function $S$ in some desired (normally low) frequency ranges, while preventing high control signals in high frequencies, which could lead to noise amplification, saturation and, in the particular case of resonant systems, the spillover phenomenon (excitation of non-modeled high frequency resonant modes).

Based on this objectives and taking into account Figure 13, the $\mathscr{H}_{\infty}$ LSDP can be described in four steps:

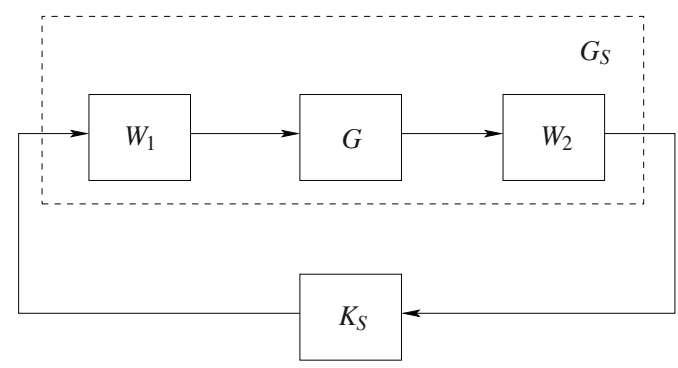

Fig. 13. Loop Shaping Design Procedure (LSDP) scheme

1) Choose the filters $W_{1}$ and $W_{2}$ to shape the desired open-loop system $G_{S}$ from the nominal model $G\left(G_{S}=\right.$ $W_{2} G W_{1}$ ). The filters are chosen accordingly to the previously mentioned principles for conforming the desired $S$ and $K S$ closed-loop functions;

2) After a normalized coprime factorization of the obtained model $G_{S}$, find the robust controller $K_{S}$ that stabilizes the closed-loop system for the largest possible set of uncertainties (associated to a stability margin $\varepsilon$ ) acting on the coprime factors [23]. This is done by solving the $\mathscr{H}_{\infty}$ problem:

$$
\min _{K}\left\|\left[\begin{array}{l}
W_{1}^{-1} \\
W_{2} G
\end{array}\right](I-K G)^{-1}\left[\begin{array}{ll}
W_{1} & G W_{2}^{-1}
\end{array}\right]\right\|_{\infty}
$$

3) If the resulting stability margin $\varepsilon$ is greater than 0.2 , the frequency response of $K_{S} W_{2} G W_{1}$ is close to that of $W_{2} G W_{1}$ [23], and we can conclude that our shaped function $G_{S}$ is close to the open-loop gain $K_{S} W_{2} G W_{1}$;

4) In this case, the controller $K$ of the original system can be recovered as $K=W_{1} K_{S} W_{2}$.

\section{B. Controller design and experimental validation}

As we are dealing with a SISO system, only the $W_{1}$ filter is considered for the $G_{S}$ shaping in the LSDP (the $W_{2}$ filter can be set to 1). The $G_{S}$ function is shaped to favour both high gains at low frequencies, implying a reduced $S$ at these frequencies, and a decreasing slope at the higher frequencies, to prevent high amplitude signals and the spillover effect. The $W_{1}$ filter is chosen as

$$
W_{1}=\frac{0.055(s+300)(s+1600)^{2}}{(s+100)^{3}}
$$

and its effect on the nominal mod ctrl $2.5 \mathrm{~kg}$ model is shown in Figure 14.

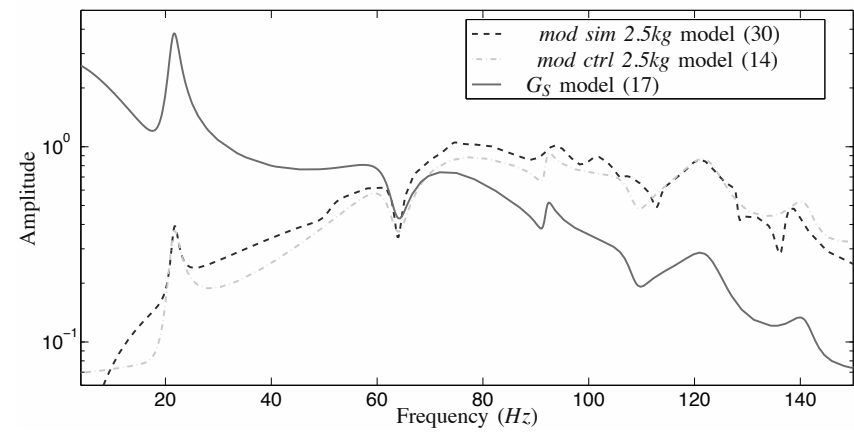

Fig. 14. LSDP open-loop ponderation

Applying the LSDP, a controller $K_{A}$ of order 19 is obtained. This controller is further reduced to a 4 order controller, named $K_{A r}$, by using a balanced realization followed by a singular perturbation reduction method. It is defined by

$$
K_{A r}=\frac{-0.44\left(s^{2}+9 s+1.32 e 4\right)\left(s^{2}-232 s+4.2 e 5\right)}{\left(s^{2}+159 s+6987\right)\left(s^{2}+29.6 s+1.48 e 4\right)}
$$

The comparison between $K_{A}$ and $K_{A r}$ is illustrated in Figure 15 , showing a good correspondence in the desired frequency range.

The sensitivity function $S_{A r}$ for the nominal simulation model ( $\bmod \operatorname{sim} 2.5 \mathrm{~kg}$ ) with the reduced order controller $K_{A r}$ is given in Figure 16, showing that frequencies around 21.7 $\mathrm{Hz}$ (corresponding to the main $Y$ direction disturbance mode shown in Figure 5) should be well attenuated. 


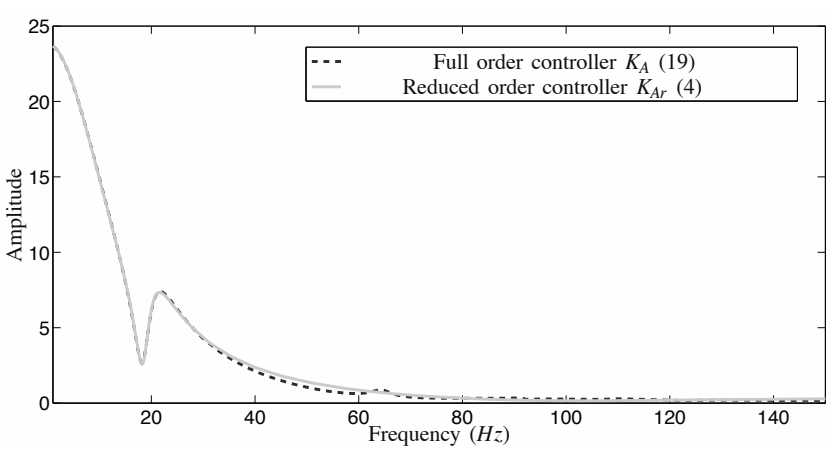

Fig. 15. Complete and reduced order controllers

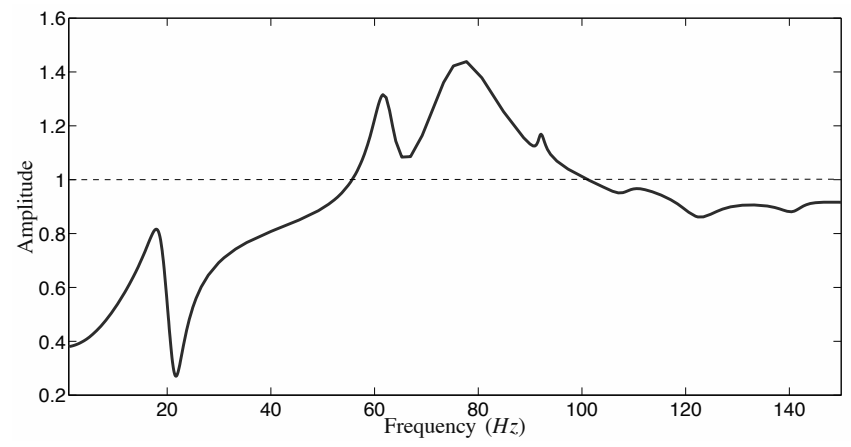

Fig. 16. Sensitivity function $S_{A r}-\bmod \operatorname{sim} 2.5 \mathrm{~kg}$ model and reduced order controller $K_{A r}$

The frequency spectrum of the open-loop response for the nominal operating point is compared in Figure 17 with the spectra obtained in closed-loop numerical simulation and experimental results. The noticeable vibration attenuation associated to the good correspondence between simulation and experimental result attest the efficiency of the proposed controller for the nominal operating point. Accordingly, time domain responses and control signals are illustrated in Figures 18 and 19.

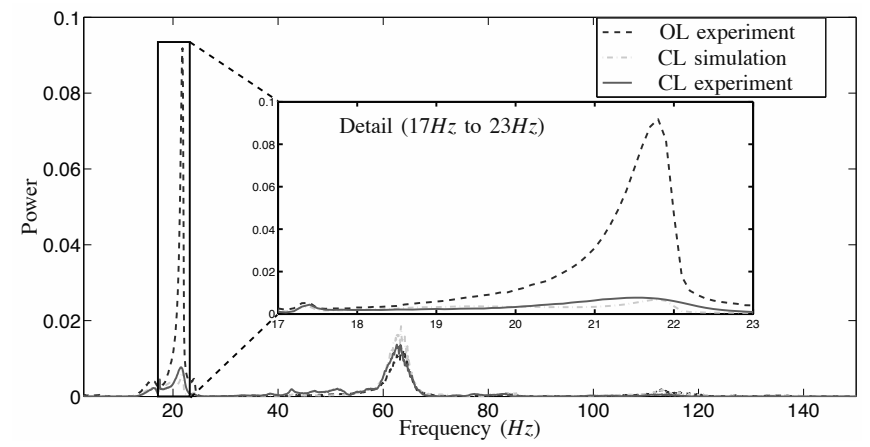

Fig. 17. Experimental results - Robot with $2.5 \mathrm{~kg}$ and reduced controller $K_{A r}-18 g$ trajectory - Power spectra

We consider now the experimental validation of the controller $K_{A r}$ applied to all operating points of Figure 4. The performance criterium adopted to measure attenuation is based on the RMS acceleration value [2]. The percentage reduction

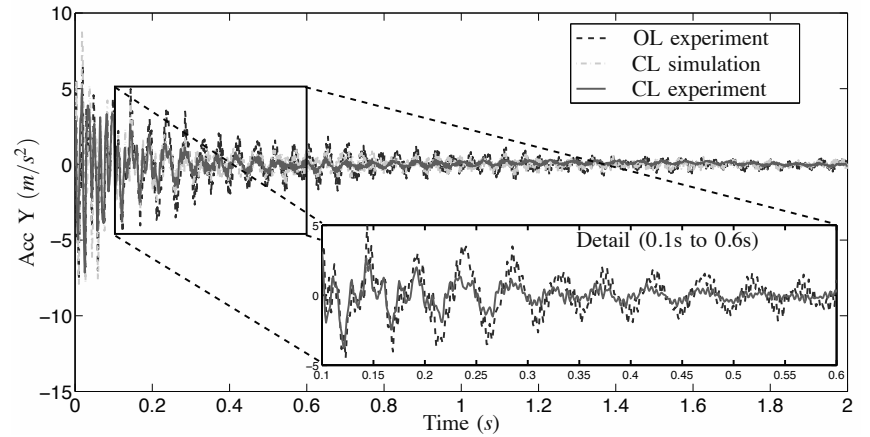

Fig. 18. Experimental results - Robot with $2.5 \mathrm{~kg}$ and reduced controller $K_{A r}-18 g$ trajectory - Simulated and experimental responses

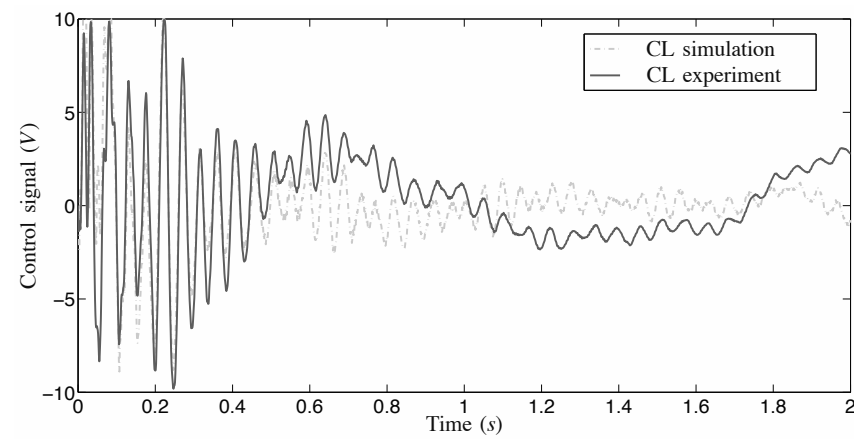

Fig. 19. Experimental results - Robot with $2.5 \mathrm{~kg}$ and reduced controller $K_{A r}-18 g$ trajectory - Control signal

of vibrations (during the first two seconds immediately after reaching stop position Pos2) in the $Y$ direction of the closedloop RMS acceleration with respect to the open-loop RMS acceleration is illustrated in Figure 20.

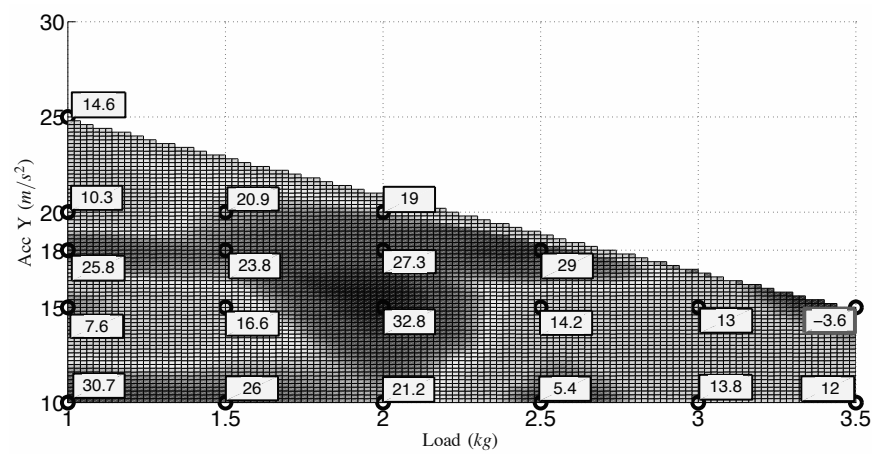

Fig. 20. Experimental results - Percentual RMS acceleration reduction during the first 2 seconds - Closed-loop system with controller $K_{A r}$ in relation to the open-loop system

From Figure 20 we can observe that the closed-loop responses with the controller $K_{A r}$ are better attenuated than those in open-loop for all but two cases. These exceptional limit cases correspond, first, to the system with a $3.5 \mathrm{~kg}$ load and a trajectory of $15 \mathrm{~g}$ (representing a $3.6 \%$ closed-loop vibration augmentation), and second, to all situations without load. This second limit case is not even represented because the controller brings the system to instability for all trajectories. The two 
limit cases have their time responses respectively represented in Figures 21 and 22. We can observe in both figures the important saturation levels leading, in the first limit case, to performance degradation and, in the second limit case, to system instabilities (illustrated for $10 \mathrm{~g}, 20 \mathrm{~g}$ and $30 \mathrm{~g}$ ).
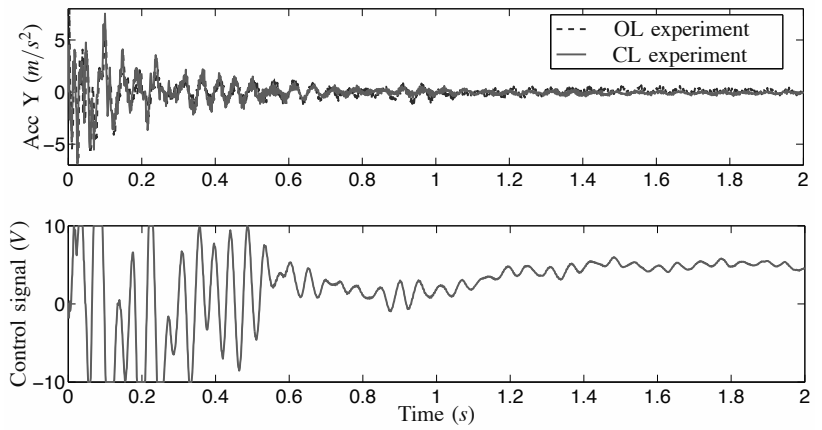

Fig. 21. Experimental results - Robot with $3.5 \mathrm{~kg}$ and controller $K_{A r}-15 \mathrm{~g}$ trajectory - Time response and control signal
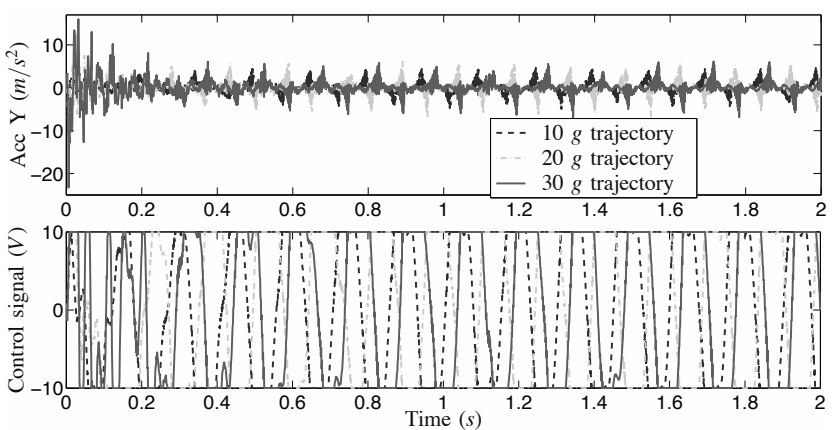

Fig. 22. Experimental results - Robot without load and controller $K_{A r}-10$ $g, 20 \mathrm{~g}$ and $30 \mathrm{~g}$ trajectories - Time response and control signal

\section{SATURATION ISSUES AND ANTI-WINDUP COMPENSATION DESIGN}

The experimental results obtained with the controller $K_{A r}$ have brought out that, despite attenuating the disturbances for almost all chosen operating points, there are still some remaining limit cases where this controller is not efficient. The stability and performance problems for these limit cases are associated with the high control signals delivered by the controller, which induce saturation of the actuator. This can also be exhibited thanks to a stability analysis of the closed-loop saturated system in presence of exogenous energy-bounded disturbance (see [32], Chapter 3, Proposition 3.14), considering explicitly that the input of the piezo-actuator model is subjected to amplitude saturation $-u_{0} \leq u(t) \leq u_{0}, \quad u_{0}=10 \mathrm{~V}$. For an a priori given bound on the disturbance, i.e., for a disturbance belonging to the set $^{1}$

$$
\mathscr{W}=\left\{w:[0, \infty) \rightarrow \mathfrak{R}^{q} ; \int_{0}^{\infty} w(\tau)^{\prime} w(\tau) d \tau \leq \delta^{-1}\right\}
$$

\footnotetext{
${ }^{1} x^{\prime}$ denotes the transpose of vector $x$.
}

one can evaluate the $\mathscr{L}_{2}$ performance gain $\gamma$ between the disturbance and the output acceleration of the end-effector. Results are summarized in Table II, both for the saturationfree case (second column) and for the saturated input case (third column).

We can check that, as expected, the controller $K_{A r}$, designed without taking saturations into account explicitly, ensures excellent results for the nominal model in the unconstrained case $(\gamma=0.08)$. As the actuator is actually subject to saturations, the saturated case (third column) exhibits that, for the nominal case, vibrations should not be amplified $(\gamma=1.00)$ but the expected performance is much reduced. When the load is changed (reduced to $2 \mathrm{~kg}$ or increased to $3.5 \mathrm{~kg}$ ), the expected performance is degraded as well for the unconstrained case and for the saturated case. This is observed experimentally, i.e. the disturbance is not attenuated but amplified in the higher load case (Figure 21). Moreover, the controller $K_{A r}$ is definitively not appropriate when the robot does not carry any load $(\gamma=165.27$ without limitation and the problem is even infeasible in presence of limitations of the actuator). This explains the loss of stability experimentally observed (Figure 22) in that case.

At that point, one option may be to design another controller with the LSDP such as to reduce the control signals and avoid, as much as possible, saturation of the piezoelectric actuator. This is for example the case of controller $K_{B r}$, designed with the $W_{1}$ weighting filter selected such as to reduce the gain from 0.2 to 0.1 . Such a solution however also reduces the disturbance attenuation performance around the nominal operation point (see Table II, column 5, where the nominal attenuation at $2.5 \mathrm{~kg}$ is increased from 0.08 with $K_{A r}$ to 0.23 with $K_{B r}$ ). This is also illustrated in Figure 23, which exhibits that the vibration attenuation with the second controller is increased with respect to the open-loop behavior for all the operating points of Figure 4, even for the limit cases, but is decreased at (and around) the nominal operating point for which have been designed the controllers.

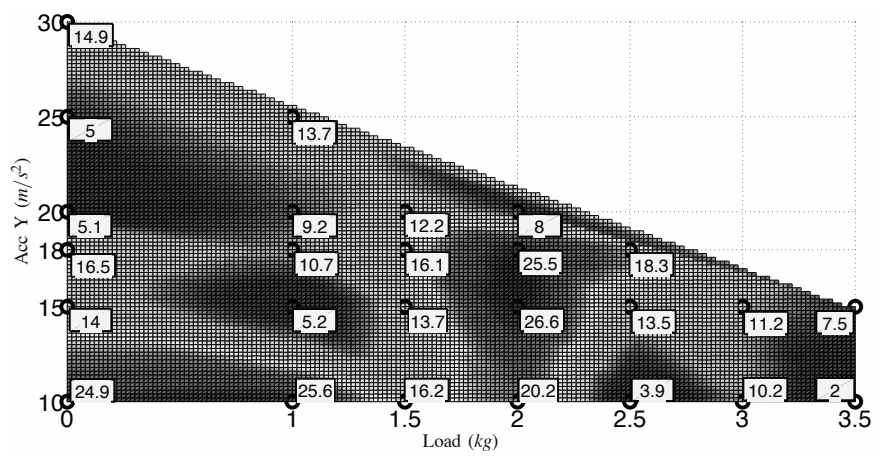

Fig. 23. Experimental results - Percentual RMS acceleration reduction during the first 2 seconds - Closed-loop system with the controller $K_{B r}$ in relation to the open-loop system

As an alternative, we propose to build an additional antiwindup compensator, which does not modify the performance of the nominal controller when no saturation occurs and only acts when the saturation becomes active. A similar anti-windup strategy has been already considered for active vibration 
isolation systems in a slightly different problem [33], and proved its efficiency.

The anti-windup strategy employed in this paper is fully described in [32] (Chapter 7, Proposition 7.4) and briefly commented here. Let us go back to Figure 7 and consider now an anti-windup compensator block acting on the controller $K_{A r}(s)$. The state-space dynamics controller $K_{A r}(s)$, between the acceleration $y$ of the end-effector (input of the controller) and the controller output $y_{K}$ is then modified as follows:

$$
\left\{\begin{array}{l}
\dot{x}_{K}(t)=A_{K} x_{K}(t)+B_{K} y(t)+v_{x}(t) \\
y_{K}(t)=C_{K} x_{K}(t)+D_{K} y(t)
\end{array}\right.
$$

where the additional input $v_{x}(t)$ allows to introduce the controller's anti-windup correction factor in the dynamics of the controller. In a static strategy, it is defined as

$$
v_{x}(t)=D_{a w}^{x}\left(u(t)-y_{K}(t)\right)
$$

i.e., it uses the error between the real input $u(t)=\operatorname{sat}\left(y_{K}(t)\right)$ and the output of the controller $y_{K}(t)$ to compute the antiwindup action. Considering the piezo-actuator model identified with a $3.5 \mathrm{~kg}$ load, the nominal controller $K_{A r}$, the disturbance bound $\delta=0.2$ and the bound $u_{0}=10$, one computes the static anti-windup compensator

$$
D_{a w}^{x}=\left[\begin{array}{llll}
-0.3032 & -0.1134 & -0.0368 & -0.1694
\end{array}\right]^{\prime}
$$

Roughly speaking, the associated $\mathscr{L}_{2}$ performance gain $\gamma$ is improved (Table II, column 4) with respect to the saturated case without anti-windup (Table II, column 3), although the values remain larger than one. It may be checked in Figure 24 that the anti-windup closed-loop system now attenuates the vibration in this operation point $(3.5 \mathrm{~kg}$ load and $15 \mathrm{~g}$ acceleration).

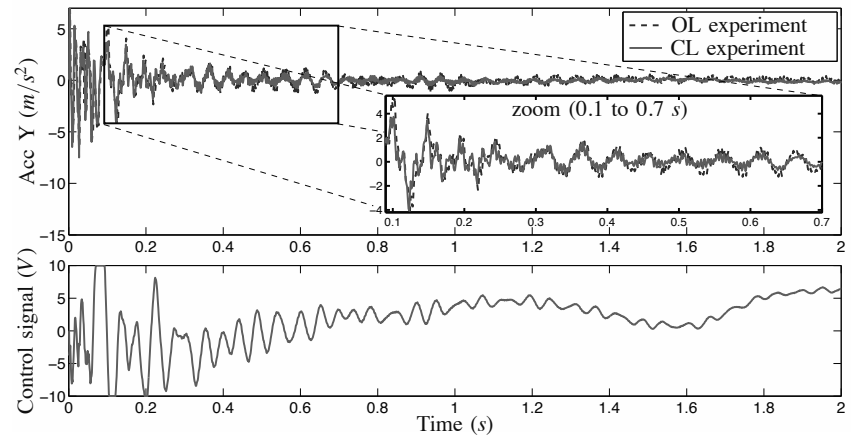

Fig. 24. Experimental results - Robot with $3.5 \mathrm{~kg}$ and controller $K_{A r}+$ anti-windup - $15 \mathrm{~g}$ trajectory - Time response and control signal

To go further, one can check that, with the same antiwindup gain, vibrations are also attenuated in the case of the robot without load (see Figure 25). Moreover, using this solution where the anti-windup action is only active when the input saturates, the vibration attenuation is unchanged for all the other operating points where the actuator almost never saturated.
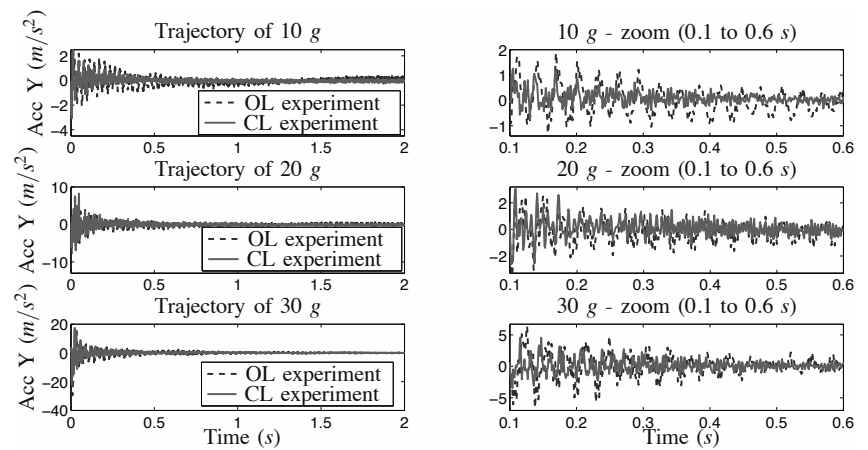

Fig. 25. Experimental results - Robot without load and controller $K_{A r}+$ anti-windup - $10 \mathrm{~g}, 20 \mathrm{~g}$ and $30 \mathrm{~g}$ trajectories - Time response and control signal

\section{CONCLUSIONS AND FUTURE WORKS}

This work presented the results obtained for the piezoactuated robust vibration attenuation of a high-speed and high-accuracy pick-and-place parallel robot in a set operating pointsdescribed by the peak accelerations and loads and for a given configuration of the stop position.

First, based on well reproducible experimental data, the N4SID subspace identification algorithm was used to estimate models for the piezo-actuator in a nominal operating point. A controller was synthesized by employing the $\mathscr{H}_{\infty}$ Loop Shaping Design Procedure to the nominal model. This controller was capable of attenuating the vibrations for almost all operating points. In the other operating points (limit cases), the high control signals saturated the system input, leading, as a consequence, to loss of performance and, in some cases, even to loss of stability. Then, rather than designing another less active controller able to prevent, as much as possible, saturating signals, we have added a static anti-windup compensator to alleviate the malicious effect of saturation. Fair performance level has been observed in such a case in experimental evaluations on the whole domain of operation.

The results obtained underline the feasibility of such strategy involving piezoelectric patches on the arms of the robot for vibration attenuation during high-speed pick-and-place movements of the parallel robot. They pave the way for future evaluation of the strategy from an industrial point-of-view, in a MIMO context and for different configurations of the stop point. Actually, if the robot configuration at the stop point is changed, then one can expect that the model will not be any more valid. Either one can design a controller for each possible configuration Pos2 (if the robot is used within a limited and a priori given set of configurations) or the control design has to be robust to the set of configurations.

\section{ACKNOWLEDGMENTS}

This work was supported in part by the French National Research Agency (ANR), in the framework of the Objectif $100 G$ project. The authors also gratefully acknowledge the reviewers for their fruitful comments. 


\section{REFERENCES}

[1] S. Algermissen, R. Keimer, M. Rose, E. Breitbach, and H.P. Monner. Applied robust control for vibration suppression in parallel robots. In Proceedings of 22nd International Symposium on Automation and Robotics in Construction (ISARC), 2005.

[2] G. J. Balas. Robust control of flexible structures: theory and experiments. $\mathrm{PhD}$ thesis, California Institute of Technology, 1990.

[3] C. Baradat, V. Nabat, O. Company, S. Krut, and F. Pierrot. Par2: a spatial mechanism for fast planar, 2-DOF, pick-and-place applications. In Proceedings of the Second International Workshop on Fundamental Issues an Future Research Directions for Parallel Mechanisms and Manipulators, pages 1-10, 2008.

[4] A. Chiuso and G. Picci. Some algorithmic aspects of subspace identification with inputs. Int. J. Appl. Math. Comput. Sci., 11(1):55-75, 2001.

[5] O. Company and F. Pierrot. Modelling and preliminary design issues of a 3-axis parallel machine-tool. Mechanisms and Machine Theory, pages $1325-1245,2002$

[6] D. Constantinescu and E. A. Croft. Smooth and time optimal trajectory planing for industrial manipulators along specified paths. Journal of Robotic Systems, 17(5):233-249, 2000.

[7] M. M. da Silva, L. P. R. de Oliveira, O. Brüls, M. Michelin, C. Baradat, O. Tempier, J. De Caigny, J. Swevers, W. Desmet, and H. Van Brussel. Integrating structural and input design of a 2-DOF high-speed parallel manipulator: a flexible model-based approach. Mechanisms and Machine Theory, 45:1509-1519, 2010.

[8] L.R. Douat, I. Queinnec, G. Garcia, M. Michelin, and F. Pierrot. Flexible model identification of the parallel robot Par2. In IEEE/RSJ Int. Conf. Intel. Robotics Systems (IROS), 2010.

[9] L.R. Douat, I. Queinnec, G. Garcia, M. Michelin, and F. Pierrot. $H_{\infty}$ control applied to the vibration minimization of the parallel robot Par2. In EEE International Conference on Control Applications (CCA), volume 947-952, Denver, USA, 2011.

[10] L.R. Douat, I. Queinnec, G. Garcia, M. Michelin, and F. Pierrot. Piezoactuated vibration attenuation of the parallel robot Par2. In IFAC Symposium on Robust Control Design (ROCOND), pages 115-120, Aalborg, DK, 2012.

[11] A. Gasparetto, A. Lanzutti, R. Vidoni, and V. Zanotto. Experimental validation and comparative analysis of optimal time-jerk algorithms for trajectory planning. Robotics and Computer-Integrated Manufacturing, 28(2):164-181, 2012.

[12] D. Gu, P. Petkov, and M. Konstantinov. Robust Control Design with MAT LAB ${ }^{\circledR}$. Springer, 2005.

[13] K. Gurses, B. J. Buckham, and E. J. Park. Vibration control of a singlelink flexible manipulator using an array of fiber optic curvature sensors and pzt actuators. Mechatronics, 19(2):167-177, 2009.

[14] M. Hassan, R. Dubay, C. Li, and R. Wang. Active vibration control of a flexible one-link manipulator using a multivariable predictive controller. Mechatronics, 17(6):311-323, 2007.

[15] N. Jalili. Piezoelectric-based vibration control: from macro to micro/nano scale systems. Springer, 2009.

[16] T. Katayama. Subspace methods for system identification. Springer, 2009.

[17] W.E. Larimore. System identification, reduced-order filtering and modeling via canonical variate analysis. In American Control Conference, pages 445-451, 1983.

[18] W.E. Larimore. Canonical variate analysis in identification, filtering and adaptive control. In Proc. 29th IEEE Conference on Decision and Control, pages 596-604, 1990.

[19] K. K. Leang and S. Devasia. Feedback-linearized inverse feedforward for creep, hysteresis, and vibration compensation in AFM piezoactuators. IEEE Trans. Control Systems Technol., 15(5):927-935, 2007.

[20] L. Ljung. System identification - Theory for the user. Prentice Hall, Upper Saddle River, NJ, 1999.

[21] A. De Luca and W. Book. Handbook of Robotics. Springer, 2008.

[22] F. Marquet, O. Company, S. Krut, and F. Pierrot. Enhancing parallel robots accuracy with redundant sensors. In IEEE Int. Conf. on Robotics and Automation - ICRA, pages 4114-4119, 2002.

[23] D. McFarlane and K. Glover. A loop shaping design procedure using $H_{\infty}$ synthesis. IEEE Trans. Automat Control, 37(6):759-769, 1992.

[24] J.-P. Merlet. Parallel Robots. Kluwer Academic Publishers, 2000.

[25] S. O. R. Moheimani and A. J. Fleming. Piezoelectric transducers for vibration control and damping. Springer, 2006.

[26] T. Nestorovic-Trajkov and U. Gabbert. Active control of a piezoelectric funnel-shaped structure based on subspace identification. Structural Control and Health Monitoring, 13(6):1068-1079, 2006.
[27] A. V. Oppenheim, A. S. Willsky, and I. T. Young. Signals and Systems. Prentice Hall, 1983.

[28] P. Van Overschee and B. DeMoor. N4SID: Subspace algorithms for the identification of combined deterministic-stochastic systems. Automatica, 30:75-93, 1994.

[29] A. Preumont. Vibration control of active structures - an introduction. Kluwer Academic Publishers, 2nd edition edition, 2002.

[30] A. C. Qiu, J. D. Han, X. M. Zhang, Y. C. Wang, and Z. W. Wu. Active vibration control of a flexible beam using a non-collocated acceleration sensor and piezoelectric patch actuator. Journal of Sound and Vibration, 326(438-455), 2009.

[31] H. Sumiya and K. Akaqi. Active damping using N4SID model referenced predictive anti-phase pitch driving method. Applied Mechanics and Materials, 36(436-441), 2010.

[32] S. Tarbouriech, G. Garcia, J.M. Gomes da Silva Jr., and I. Queinnec. Stability and stabilization of linear systems with saturating actuators. Springer-Verlag, 2011.

[33] A.R. Teel, L. Zaccarian, and J.J. Marcinkowski. An anti-windup strategy for active vibration isolation systems. Control Engineering Practice, 14:17-27, 2006

[34] S. Tliba, H. Abou-Kandil, and C. Prieur. Active vibration damping of a smart flexible structure using piezoelectric transducers: H-infinity design and experimental results. In Proceedings of the 16th IFAC World Congress, 2005.

[35] M. Verhaegen. Identification of the deterministic part of MIMO state space models given in innovations form from input-output data. Automatica, 29(4):61-74, 1994.

[36] M. Verhaegen and V. Verdult. Filtering and system identification: An introduction. Lecture notes for the course sc4040 (et4094), Delft, 2003

[37] S.L.H. Verhoeven. Robust control of flexible motion systems: A literature study. Technical report, DCT Report 2009.006, APT536-6288, 2009.

[38] M. Viberg. On subspace-based methods for the identification of linear time-invariant systems. Automatica, 31(12):1835-1852, 1995.

[39] L. Zaccarian and A.R. Teel. Modern anti-windup synthesis. Princeton University Press, 2011

[40] K. Zhou and J. Doyle. Essentials of Robust Control. Prentice Hall, 1998. 
TABLE II

Performance ANALysis of THE ClOSED-LOOP System (FOR $\delta=0.2$ )

\begin{tabular}{|c|c|c|c|c|}
\hline & \multicolumn{3}{|c|}{ controller $K_{A r}$} & controller $K_{B r}$ \\
\hline model & $\begin{array}{c}\gamma \\
\text { unconstrained }\end{array}$ & $\begin{array}{c}\gamma \\
\text { with saturation }\end{array}$ & $\begin{array}{c}\gamma \\
\text { with saturation and AW }\end{array}$ & $\begin{array}{c}\gamma \\
\text { unconstrained }\end{array}$ \\
\hline empty & 165.27 & unfeasible & $\begin{array}{ll}\text { unfeasible } \\
\end{array}$ & 77.72 \\
\hline $2 \mathrm{~kg}$ & 0.52 & 1.98 & 1.09 & 0.77 \\
\hline $2.5 \mathrm{~kg}$ & 0.08 & 1.00 & 0.75 & 0.23 \\
\hline $3.5 \mathrm{~kg}$ & 0.80 & 1.97 & 1.45 & 1.02 \\
\hline
\end{tabular}

